\title{
Centralized and Decentralized Data-Sampling Principles for Outer-Synchronization of Fractional-Order Neural Networks
}

\author{
Jin-E Zhang \\ Hubei Normal University, Hubei 435002, China \\ Correspondence should be addressed to Jin-E Zhang; zhang86021205@163.com \\ Received 24 December 2016; Revised 6 February 2017; Accepted 21 February 2017; Published 8 March 2017 \\ Academic Editor: Olfa Boubaker \\ Copyright (C) 2017 Jin-E Zhang. This is an open access article distributed under the Creative Commons Attribution License, which \\ permits unrestricted use, distribution, and reproduction in any medium, provided the original work is properly cited.

\begin{abstract}
This paper aims to investigate the outer-synchronization of fractional-order neural networks. Using centralized and decentralized data-sampling principles and the theory of fractional differential equations, sufficient criteria about outer-synchronization of the controlled fractional-order neural networks are derived for structure-dependent centralized data-sampling, state-dependent centralized data-sampling, and state-dependent decentralized data-sampling, respectively. A numerical example is also given to illustrate the superiority of theoretical results.
\end{abstract}

\section{Introduction}

Fractional operator has become visible in application domains [1-15]. As the demanding performance expectations with uncertainty, fractional operator offers more degrees of freedom to designers to meet some predefined performance indexes. After gradually recognizing the importance of fractional operator, it is found that the description of fractional-order model is more accurate and totally different from that of the corresponding integer-order model. As a direct application, the characteristic of fractional-order model can be used to identify possible behavior of electrical signals from neurons. In physical implementation of neurodynamic systems, arbitrary order analog fractance circuit is most appropriate, which reveals profoundly the relationships among neural circuit elements [9-11]. In that way, real neurodynamic systems should be addressed by fractional-order models. Fractional-order neurodynamic systems can better describe how action potentials in neurons are launched and spread. In addition, fractional-order neurodynamic systems possess infinite memory, and yet, integer-order neurodynamic systems are not of such feature $[3-8,12-15]$. Therefore, fractional-order neurodynamic systems have the potential to accomplish what integerorder ones can not do. More feasible analysis methods and easy-to-use techniques to be deal with fractional-order neurodynamic systems are worth looking into.

As a coherent behavior within nonlinear systems, synchronization of nonlinear systems has attracted phenomenal worldwide attention. Many studies have shown that synchronization mechanism is a universal phenomenon and has a wide range of applications in engineering systems. Generally, two schemes for synchronization are frequently used: inner-synchronization and outer-synchronization. For innersynchronization, all nodes within a network will achieve a coherent behavior. However, for outer-synchronization, all individuals in two networks will achieve identical behaviors. In many application fields, outer-synchronization may seem practical [16-23]. For example, in heuristic computational intelligence, it is known that outer-synchronization is rooted in brain-inspired computing from evolutionary strategies to cognitive tasks. Nevertheless, results focusing on outer-synchronization of complex control systems have seldom been reported [19]. Control strategy for outersynchronization deserves more investigation.

Sampled-data control through only using the local information has recently generated significant research interest [24-38]. Unlike continuous-time control, which requires the continuous communication data, sampled-data control is more appropriate under networked environment. For 
control systems, once we can give effective sampling policies and schedule, then the sampled-data control will reduce communication data and save energy dramatically. Thus, how to develop high-efficiency, heuristic information-based sampled-data control with the ultimate aim of maximizing the data collected is worth studying [38]. However, relevant studies of the data-sampling strategy for control systems are still in early stage.

Motivated by the above discussions, in this paper, we introduce the centralized and decentralized data-sampling principles to achieve outer-synchronization between coupled fractional-order neural networks. The efficient allocation of the limited energy resources of centralized and decentralized data-sampling principles that maximizes the information value of the data collected is clearly a step forward. Meanwhile, to more efficiently design the sampling method, we merge the structure and state clusters through centralized and decentralized data-sampling principles and then select the best sampling time. On the basis of some analytical tools of fractional differential equations, a series of criteria on outersynchronization are derived. It should be noted that such criteria capture the information on sampling pattern and may have much wider application range.

The rest of the paper is organized as follows. In Section 2, we present the preliminaries and problem formulation. In Section 3, we state main results in detail. In Section 4, simulation example is illustrated. Finally, Section 5 concludes the paper.

\section{Preliminaries and Problem Formulation}

First, some preliminaries of fractional operator are given.

Fractional integral $I^{q}(\cdot)$ for $\mathscr{H}(t)$ with order $q>0$ is described as

$$
I^{q} \mathscr{H}(t)=\frac{1}{\Gamma(q)} \int_{t_{0}}^{t}(t-s)^{q-1} \mathscr{H}(s) d s, \quad t \geq t_{0},
$$

where $\Gamma(\cdot)$ is Gamma function and $t_{0}$ is the initial time.

Caputo fractional derivative ${ }^{C} D_{t_{0}}^{q}(\cdot)$ for $\mathscr{H}(t)$ $\mathscr{C}^{m+1}\left(\left[t_{0},+\infty\right), \mathfrak{R}\right)$ with order $q>0$ is described as

$$
{ }^{C} D_{t_{0}}^{q} \mathscr{H}(t)=\frac{1}{\Gamma(m-q)} \int_{t_{0}}^{t} \frac{\mathscr{H}^{(m)}(s)}{(t-s)^{q-m+1}} d s, \quad t \geq t_{0},
$$

where $\Gamma(\cdot)$ is Gamma function, $m-1<q<m, m$ is a positive integer, and $t_{0}$ is the initial time.

One-parameter Mittag-Leffler function $E_{q}(\cdot)$ is described as

$$
E_{q}(s)=\sum_{k=0}^{+\infty} \frac{s^{k}}{\Gamma(k q+1)},
$$

where $\Gamma(\cdot)$ is Gamma function, $q>0$, and $s$ is a complex number.

Consider a class of fractional-order neural networks

$$
\begin{aligned}
{ }^{C} D_{t_{0}}^{q} x_{i}(t)= & -a_{i}(t) x_{i}(t)+\sum_{j=1}^{n} b_{i j}(t) f_{j}\left(x_{j}(t)\right) \\
& +u_{i}(t), \quad i=1,2, \ldots, n,
\end{aligned}
$$

where $0<q<1, a_{i}(t)>0, b_{i j}(t)$ and $u_{i}(t)$ are piecewise continuous and bounded, and feedback function $f_{j}(\cdot)$ satisfies

$$
\begin{aligned}
& 0 \leq \frac{f_{j}\left(\mathscr{U}_{1}\right)-f_{j}\left(\mathscr{U}_{2}\right)}{\mathscr{U}_{1}-\mathscr{U}_{2}} \leq F_{j}, \\
& \forall \mathscr{U}_{1}, \mathscr{U}_{2} \in \mathfrak{R}, \mathscr{U}_{1} \neq \mathscr{U}_{2},
\end{aligned}
$$

in which $F_{j}>0, j=1,2, \ldots, n$.

For the centralized data-sampling principle, (4) is rewritten as

$$
\begin{aligned}
{ }^{C} D_{t_{0}}^{q} x_{i}(t)= & -a_{i}(t) x_{i}\left(t_{k}\right)+\sum_{j=1}^{n} b_{i j}(t) f_{j}\left(x_{j}\left(t_{k}\right)\right) \\
& +u_{i}(t), \quad i=1,2, \ldots, n,
\end{aligned}
$$

where $t_{k}$ is simple notion of $t_{k(t)}$ with $k(t)=\max \left\{\mathscr{K}: t_{\mathscr{K}} \leq t\right\}$ and $0=t_{0}<t_{1}<\cdots<t_{k}<\cdots$ is uniform for all the system states. Every neuron intersperses its state to its out-neighbors and receives the state information from its in-neighbors at the same time point $t_{k}$.

For the decentralized data-sampling principle, (4) is rewritten as

$$
\begin{aligned}
{ }^{C} D_{t_{0}}^{q} x_{i}(t)= & -a_{i}(t) x_{i}\left(t_{k}^{i}\right)+\sum_{j=1}^{n} b_{i j}(t) f_{j}\left(x_{j}\left(t_{k}^{j}\right)\right) \\
& +u_{i}(t), \quad i=1,2, \ldots, n,
\end{aligned}
$$

where $t_{k}^{i}$ is simple notion of $t_{k(t)}^{i}$ with $k(t)=\max \left\{\mathscr{K}: t_{\mathscr{K}}^{i} \leq t\right\}$ and $0=t_{0}^{i}<t_{1}^{i}<\cdots<t_{k}^{i}<\cdots$ is distributed for $i \in\{1,2, \ldots, n\}$. Each neuron $i$ pushes its state information to its out-neighbors at time $t_{k}^{i}$ when it updates its state. It receives the information of in-neighbor state at time $t_{k}^{j}$ when the neighbor neuron $j$ updates its state.

Now, we state definition and problem formulation.

Definition 1 (see [19]). For any two trajectories $w(t)=$ $\left(w_{1}(t), w_{2}(t), \ldots, w_{n}(t)\right)^{T}$ and $\bar{w}(t)=\left(\bar{w}_{1}(t), \bar{w}_{2}(t), \ldots\right.$, $\left.\bar{w}_{n}(t)\right)^{T}$ of (4) starting from different initial values $w(0)$ and $\bar{w}(0)$, if there exists some control scheme such that

$$
\lim _{t \rightarrow+\infty}\|w(t)-\bar{w}(t)\|=0
$$

then we call system (4) can achieve outer-synchronization, where $\|\cdot\|$ denotes norm.

Let $u(t)=\left(u_{1}(t), u_{2}(t), \ldots, u_{n}(t)\right)^{T}$ and $v(t)=\left(v_{1}(t)\right.$, $\left.v_{2}(t), \ldots, v_{n}(t)\right)^{T}$ be two trajectories of (6) starting from different initial values $u(0)$ and $v(0)$. Defining $z_{i}(t)=u_{i}(t)-$ $v_{i}(t), i=1,2, \ldots, n$, it follows that

$$
{ }^{C} D_{t_{0}}^{q} z_{i}(t)=-a_{i}(t) z_{i}\left(t_{k}\right)+\sum_{j=1}^{n} b_{i j}(t) h_{j}\left(t_{k}\right),
$$

$$
i=1,2, \ldots, n \text {, }
$$


where $h_{j}(t)=f_{j}\left(u_{j}(t)\right)-f_{j}\left(v_{j}(t)\right), j=1,2, \ldots, n$, for all $t \in\left[t_{k}, t_{k+1}\right), k=0,1,2, \ldots$

When we adopt the centralized data-sampling principle via structure to achieve outer-synchronization of (6), according to Definition 1, we need to design control strategy based on system structure of (9) such that

$$
\lim _{t \rightarrow+\infty}\|z(t)\|=0
$$

where $\|\cdot\|$ denotes norm, $z(t)=\left(z_{1}(t), z_{2}(t), \ldots, z_{n}(t)\right)^{T}$.

When we adopt the centralized data-sampling principle via state to achieve outer-synchronization of (6), in this case, consider state measurement error

$$
e_{i}(t)=z_{i}\left(t_{k}\right)-z_{i}(t), \quad i=1,2, \ldots, n,
$$

where $t \in\left[t_{k}, t_{k+1}\right), k=0,1,2, \ldots$ According to Definition 1, we need to design control strategy based on state measurement error (11) such that

$$
\lim _{t \rightarrow+\infty}\|z(t)\|=0
$$

where $\|\cdot\|$ denotes norm, $z(t)=\left(z_{1}(t), z_{2}(t), \ldots, z_{n}(t)\right)^{T}$.

Let $u(t)=\left(u_{1}(t), u_{2}(t), \ldots, u_{n}(t)\right)^{T}$ and $v(t)=$ $\left(v_{1}(t), v_{2}(t), \ldots, v_{n}(t)\right)^{T}$ be two trajectories of (7) starting from different initial values $u(0)$ and $v(0)$. Defining $z_{i}(t)=$ $u_{i}(t)-v_{i}(t), i=1,2, \ldots, n$, it follows that

$$
\begin{array}{r}
{ }^{C} D_{t_{0}}^{q} z_{i}(t)=-a_{i}(t) z_{i}\left(t_{k}^{i}\right)+\sum_{j=1}^{n} b_{i j}(t) h_{j}\left(t_{k}^{j}\right), \\
\quad i=1,2, \ldots, n,
\end{array}
$$

where $h_{j}(t)=f_{j}\left(u_{j}(t)\right)-f_{j}\left(v_{j}(t)\right), j=1,2, \ldots, n$, for all $t \in\left[t_{k}^{j}, t_{k+1}^{j}\right), k=0,1,2, \ldots$

When we adopt the decentralized data-sampling principle via state to achieve outer-synchronization of (7), in this case, consider state measurement error

$$
e_{i}(t)=z_{i}\left(t_{k}^{i}\right)-z_{i}(t), \quad i=1,2, \ldots, n,
$$

where $t \in\left[t_{k}^{i}, t_{k+1}^{i}\right), k=0,1,2, \ldots$ According to Definition 1, we need to design control strategy based on state measurement error (14) such that

$$
\lim _{t \rightarrow+\infty}\|z(t)\|=0
$$

where $\|\cdot\|$ denotes norm, $z(t)=\left(z_{1}(t), z_{2}(t), \ldots, z_{n}(t)\right)^{T}$.

Next, we present relevant lemmas.

Lemma 2 (see [1]). Let $0<q<1$. If $\mathscr{H}(t) \in \mathscr{C}^{1}\left[t_{0},+\infty\right]$, then

$$
{ }^{C} D_{t_{0}}^{q}|\mathscr{H}(t)| \leq \operatorname{sgn}(\mathscr{H}(t)){ }^{C} D_{t_{0}}^{q} \mathscr{H}(t), \quad t \geq t_{0},
$$

where

$$
{ }^{C} D_{t_{0}}^{q}|\mathscr{H}(t)|=\frac{1}{\Gamma(1-q)} \int_{t_{0}}^{t} \frac{(d / d s)|\mathscr{H}(s)|}{(t-s)^{q}} d s .
$$

Lemma 3 (see [39]). Given $q>0$, let $\mathscr{A}(t)$ be nonnegative and locally integrable on $[a, b)$; let $\mathscr{G}(t)$ be continuous, bounded, nonnegative, and nondecreasing on $[a, b)$. Assuming $\mathcal{Y}(t)$ to be nonnegative and locally integrable on $[a, b)$ with

$$
\mathscr{Y}(t) \leq \mathscr{A}(t)+\mathscr{G}(t) \int_{0}^{t}(t-s)^{q-1} \mathscr{Y}(s) d s
$$

$$
t \in[a, b) \text {, }
$$

then

$$
\begin{aligned}
\mathscr{Y}(t) & \\
\leq & \mathscr{A}(t) \\
& +\int_{0}^{t} \sum_{k=1}^{+\infty}\left[\frac{[\mathscr{G}(t) \Gamma(q)]^{k}}{\Gamma(k q)}(t-s)^{k q-1} \mathscr{A}(s)\right] d s, \\
& t \in[a, b) .
\end{aligned}
$$

Moreover, if $\mathscr{A}(t)$ is nondecreasing on $[a, b)$, then

$$
\mathscr{Y}(t) \leq \mathscr{A}(t) E_{q}\left(\mathscr{G}(t) \Gamma(q) t^{q}\right), \quad t \in[a, b),
$$

where $\Gamma(\cdot)$ is Gamma function and $E_{q}(\cdot)$ is one-parameter Mittag-Leffler function.

In the following, we end this section with some notations that are needed later.

Let $\beta_{i}>0(i=1,2, \ldots, n)$ be positive constants, throughout this paper; denote

$$
\begin{aligned}
\theta_{i}(t) & =a_{i}(t)-F_{i} b_{i i}^{+}(t), \\
\lambda_{j}(\beta, t) & =\theta_{j}(t)-F_{j} \sum_{j=1, j \neq i}^{n} \frac{\beta_{i}}{\beta_{j}}\left|b_{i j}(t)\right|, \\
\rho(t) & =\max _{1 \leq i \leq n}\left\{a_{i}(t)-F_{i} b_{i i}^{-}(t)\right\}, \\
M & =\max _{1 \leq j \leq n} \sup _{t \geq t_{0}}\left\{a_{j}(t)+\sum_{i=1}^{n} \frac{\beta_{i}}{\beta_{j}}\left|b_{i j}(t)\right| F_{j}\right\},
\end{aligned}
$$

where $b_{i i}^{+}(t)=\max \left\{0, b_{i i}(t)\right\}, b_{i i}^{-}(t)=\min \left\{0, b_{i i}(t)\right\}$. For vector $\mathscr{V}=\left(\mathscr{V}_{1}, \mathscr{V}_{2}, \ldots, \mathscr{V}_{n}\right)^{T}$, vector norm $\|\mathscr{V}\|=$ $\sum_{i=1}^{n} \beta_{i}\left|\mathscr{V}_{i}\right|$. In addition, by the boundedness of $a_{i}(t)$ and $b_{i j}(t)$, there exist positive constants $B$ and $C$ such that

$$
\begin{array}{r}
\sup _{t \geq t_{0}} \rho(t) \leq B, \\
\max _{1 \leq j \leq n} \sup _{t \geq t_{0}} \lambda_{j}(\beta, t) \leq C .
\end{array}
$$

\section{Main Results}

For problem formulation in preceding section, in this section, we propose the corresponding control schemes for centralized data-sampling principle and decentralized datasampling principle, respectively.

To facilitate the narrative, we first address the control designs, then review, and analyze the theoretical results. 


\subsection{Centralized Data-Sampling Principle}

Theorem 4. Let $0<\varepsilon<1$ and $\iota>0$ be positive constants with $B \varepsilon \leq \iota$ and $C \varepsilon \leq \iota(2-\varepsilon)$. Assume that there exist positive constants $\beta_{i}>0(i=1,2, \ldots, n)$ such that $\lambda_{j}(\beta, t) \geq \iota$ for all $j=1,2, \ldots, n$ and $t \in\left[t_{0},+\infty\right)$. Set $t_{k+1}$ as a time point such that

$$
\begin{aligned}
& t_{k+1} \\
& \quad=\sup _{\tau \geq t_{k}}\left\{\tau: \min _{1 \leq j \leq n}\left(\frac{1}{\Gamma(q)} \int_{t_{k}}^{t}(t-s)^{q-1} \lambda_{j}(\beta, s) d s\right)\right. \\
& \left.\quad \leq \varepsilon, \forall t \in\left(t_{k}, \tau\right]\right\}
\end{aligned}
$$

for $k=0,1,2, \ldots$. Then system (6) reaches outersynchronization.

Proof. From $\lambda_{j}(\beta, t) \geq \iota$ for all $j=1,2, \ldots, n$ and $t \in$ $\left[t_{0},+\infty\right)$, together with (23), it follows that

$$
\begin{aligned}
& \frac{\iota}{\Gamma(q)} \int_{t_{k}}^{t}(t-s)^{q-1} d s \\
& \quad \leq \frac{1}{\Gamma(q)} \int_{t_{k}}^{t}(t-s)^{q-1} \lambda_{j}(\beta, s) d s \\
& \quad \leq \frac{C}{\Gamma(q)} \int_{t_{k}}^{t}(t-s)^{q-1} d s
\end{aligned}
$$

then

$$
\begin{aligned}
\frac{\iota\left(t-t_{k}\right)^{q}}{\Gamma(q+1)} & \leq \frac{1}{\Gamma(q)} \int_{t_{k}}^{t}(t-s)^{q-1} \lambda_{j}(\beta, s) d s \\
& \leq \frac{C\left(t-t_{k}\right)^{q}}{\Gamma(q+1)},
\end{aligned}
$$

for all $j=1,2, \ldots, n$ and any $t \in\left[t_{k}, t_{k+1}\right)$. According to (9), $z(t)=\left(z_{1}(t), z_{2}(t), \ldots, z_{n}(t)\right)^{T}$ will not update until

$$
\min _{1 \leq j \leq n}\left(\frac{1}{\Gamma(q)} \int_{t_{k}}^{t}(t-s)^{q-1} \lambda_{j}(\beta, s) d s\right)=\varepsilon
$$

at time point $t=t_{k+1}$. Thus, we get $\iota\left(t_{k+1}-t_{k}\right)^{q} / \Gamma(q+1) \leq \varepsilon \leq$ $C\left(t_{k+1}-t_{k}\right)^{q} / \Gamma(q+1)$, which implies

$$
\frac{\varepsilon}{C} \leq \frac{\left(t_{k+1}-t_{k}\right)^{q}}{\Gamma(q+1)} \leq \frac{\varepsilon}{l}
$$

for $k=0,1,2, \ldots$ Then

$$
t_{k} \geq t_{0}+k\left(\frac{\Gamma(q+1) \varepsilon}{C}\right)^{1 / q}
$$

therefore, the Zeno behavior can be excluded. Combining with (24) and (28),

$$
\begin{aligned}
& \frac{1}{\Gamma(q)} \int_{t_{k}}^{t_{k+1}}\left(t_{k+1}-s\right)^{q-1} \lambda_{j}(\beta, s) d s \leq \frac{C \varepsilon}{\iota} \leq 2-\varepsilon, \\
& \frac{1}{\Gamma(q)} \int_{t_{k}}^{t_{k+1}}\left(t_{k+1}-s\right)^{q-1} \lambda_{j}(\beta, s) d s \\
& \quad \geq \min _{1 \leq j \leq n} \frac{1}{\Gamma(q)} \int_{t_{k}}^{t_{k+1}}\left(t_{k+1}-s\right)^{q-1} \lambda_{j}(\beta, s) d s=\varepsilon,
\end{aligned}
$$

so

$$
\varepsilon \leq \frac{1}{\Gamma(q)} \int_{t_{k}}^{t_{k+1}}\left(t_{k+1}-s\right)^{q-1} \lambda_{j}(\beta, s) d s \leq 2-\varepsilon
$$

for $k=0,1,2, \ldots$. By the definition of vector norm in this paper, from (9), now let us consider $z_{i}(t)(i=1,2, \ldots, n)$ at time $t=t_{k+1}$,

$$
\begin{aligned}
& \left\|z\left(t_{k+1}\right)\right\|=\sum_{i=1}^{n} \beta_{i}\left|z_{i}\left(t_{k+1}\right)\right|=\sum_{i=1}^{n} \beta_{i} \mid z_{i}\left(t_{k}\right)+\frac{1}{\Gamma(q)} \\
& \cdot \int_{t_{k}}^{t_{k+1}}\left(t_{k+1}-s\right)^{q-1} \\
& \cdot\left[-a_{i}(s) z_{i}\left(t_{k}\right)+\sum_{j=1}^{n} b_{i j}(s) h_{j}\left(t_{k}\right)\right] d s \mid=\sum_{i=1}^{n} \beta_{i} \\
& \mid z_{i}\left(t_{k}\right)+\frac{1}{\Gamma(q)} \int_{t_{k}}^{t_{k+1}}\left(t_{k+1}-s\right)^{q-1}\left[-a_{i}(s) z_{i}\left(t_{k}\right)\right. \\
& +b_{i i}(s) m_{i}\left(t_{k}\right) z_{i}\left(t_{k}\right) \\
& \left.+\sum_{j=1, j \neq i}^{n} b_{i j}(s) m_{j}\left(t_{k}\right) z_{j}\left(t_{k}\right)\right] d s \mid=\sum_{i=1}^{n} \beta_{i} \\
& \cdot z_{i}\left(t_{k}\right)\left\{1-\frac{1}{\Gamma(q)} \int_{t_{k}}^{t_{k+1}}\left(t_{k+1}-s\right)^{q-1}\right. \\
& \left.\cdot\left[a_{i}(s)-b_{i i}(s) m_{i}\left(t_{k}\right)\right] d s\right\}+\frac{1}{\Gamma(q)} \\
& \sum_{j=1, j \neq i}^{n} \int_{t_{k}}^{t_{k+1}}\left(t_{k+1}-s\right)^{q-1} \\
& \cdot\left[b_{i j}(s) m_{j}\left(t_{k}\right) z_{j}\left(t_{k}\right)\right] d s \mid,
\end{aligned}
$$

where

$$
m_{i}(t)= \begin{cases}\frac{h_{i}(t)}{z_{i}(t)}, & z_{i}(t) \neq 0 \\ 0, & z_{i}(t)=0\end{cases}
$$


According to (5), obviously, $0 \leq m_{i}(t) \leq F_{i}$ for all $i=$ $1,2, \ldots, n, t>t_{0}$, and

$$
b_{i i}^{-}(s) F_{i} \leq b_{i i}(s) m_{i}\left(t_{k}\right) \leq b_{i i}^{+}(s) F_{i} .
$$

Notice that $B \varepsilon \leq \imath$; then for any $t \in\left[t_{k}, t_{k+1}\right]$,

$$
\begin{aligned}
& \frac{1}{\Gamma(q)} \int_{t_{k}}^{t_{k+1}}\left(t_{k+1}-s\right)^{q-1}\left[a_{i}(s)-b_{i i}(s) m_{i}\left(t_{k}\right)\right] d s \\
& \quad \leq \frac{1}{\Gamma(q)} \int_{t_{k}}^{t_{k+1}}\left(t_{k+1}-s\right)^{q-1}\left[a_{i}(s)-b_{i i}^{-}(s) F_{i}\right] d s \\
& \quad \leq \frac{B}{\Gamma(q)} \int_{t_{k}}^{t_{k+1}}\left(t_{k+1}-s\right)^{q-1} d s=\frac{B\left(t_{k+1}-t_{k}\right)^{q}}{\Gamma(q+1)} \\
& \quad \leq \frac{B \varepsilon}{\iota} \leq 1
\end{aligned}
$$

thus

$$
\begin{aligned}
1- & \frac{1}{\Gamma(q)} \int_{t_{k}}^{t_{k+1}}\left(t_{k+1}-s\right)^{q-1}\left[a_{i}(s)-b_{i i}(s) m_{i}\left(t_{k}\right)\right] d s \\
& \geq 0 .
\end{aligned}
$$

By (32) and (36),

$$
\begin{aligned}
& \left\|z\left(t_{k+1}\right)\right\|=\sum_{i=1}^{n} \beta_{i}\left|z_{i}\left(t_{k+1}\right)\right| \leq \sum_{i=1}^{n} \beta_{i} z_{i}\left(t_{k}\right)\left\{1-\frac{1}{\Gamma(q)}\right. \\
& \left.\cdot \int_{t_{k}}^{t_{k+1}}\left(t_{k+1}-s\right)^{q-1}\left[a_{i}(s)-b_{i i}(s) m_{i}\left(t_{k}\right)\right] d s\right\} \\
& +\frac{1}{\Gamma(q)} \sum_{i=1}^{n} \beta_{i} \\
& \cdot \sum_{j=1, j \neq i}^{n} \int_{t_{k}}^{t_{k+1}}\left(t_{k+1}-s\right)^{q-1} b_{i j}(s) m_{j}\left(t_{k}\right) z_{j}\left(t_{k}\right) d s \\
& \leq \sum_{j=1}^{n} \beta_{j}\left|z_{j}\left(t_{k}\right)\right|\left\{1-\frac{1}{\Gamma(q)}\right. \\
& \left.\cdot \int_{t_{k}}^{t_{k+1}}\left(t_{k+1}-s\right)^{q-1}\left[a_{j}(s)-b_{j j}^{+}(s) F_{j}\right] d s\right\}+\frac{1}{\Gamma(q)} \\
& \cdot \sum_{i=1}^{n} \sum_{j=1, j \neq i}^{n} \beta_{j}\left|z_{j}\left(t_{k}\right)\right| \\
& \cdot \int_{t_{k}}^{t_{k+1}}\left[\left(t_{k+1}-s\right)^{q-1} \frac{\beta_{i}}{\beta_{j}}\left|b_{i j}(s)\right| F_{j}\right] d s \\
& \leq \sum_{j=1}^{n} \beta_{j}\left|z_{j}\left(t_{k}\right)\right|\left\{1-\frac{1}{\Gamma(q)}\right. \\
& \left.\cdot \int_{t_{k}}^{t_{k+1}}\left(t_{k+1}-s\right)^{q-1} \lambda_{j}(\beta, s) d s\right\} \leq(1-\varepsilon) \\
& \cdot \sum_{j=1}^{n} \beta_{j}\left|z_{j}\left(t_{k}\right)\right|=(1-\varepsilon)\left\|z\left(t_{k}\right)\right\| \text {, }
\end{aligned}
$$

for all $k=0,1,2, \ldots$, which excludes the Zeno behavior for rule (24).

Theorem 6. Let $\varphi(t)$ be a positive and continuous function on $\left[t_{0},+\infty\right)$. Set $t_{k+1}$ as a time point such that

$$
t_{k+1}=\sup _{\tau \geq t_{k}}\left\{\tau:\|e(t)\| \leq \varphi(t), \forall t \in\left(t_{k}, \tau\right]\right\}
$$

for all $k=0,1,2, \ldots$, where $e(t)=\left(e_{1}(t), e_{2}(t), \ldots, e_{n}(t)\right)^{T}$ is defined in (11). If there exist positive constants $\beta_{i}>0(i=$ $1,2, \ldots, n)$ such that $\min _{1 \leq j \leq n} \lambda_{j}(\beta, t) \geq N$ for some $N>0$ and all $t \geq t_{0}, \sup _{t \geq t_{0}}(1 / \Gamma(q)) \int_{t_{0}}^{t}(t-s)^{q-1} \varphi(s) d s<+\infty$, then system (6) reaches outer-synchronization. 
Proof. According to Lemma 2, from (9) and (42),

$$
\begin{aligned}
& { }^{C} D_{t_{k}}^{q}\|z(t)\|=\sum_{i=1}^{n} \beta_{i}{ }^{C} D_{t_{k}}^{q}\left|z_{i}(t)\right| \leq \sum_{i=1}^{n} \operatorname{sgn}\left(z_{i}(t)\right) \\
& \cdot \beta_{i}{ }^{C} D_{t_{k}}^{q} z_{i}(t)=\sum_{i=1}^{n} \operatorname{sgn}\left(z_{i}(t)\right) \beta_{i}\left\{-a_{i}(t) z_{i}\left(t_{k}\right)\right. \\
& \left.+\sum_{j=1}^{n} b_{i j}(t) h_{j}\left(t_{k}\right)\right\}=\sum_{i=1}^{n} \operatorname{sgn}\left(z_{i}(t)\right) \beta_{i}\left\{-a_{i}(t)\right. \\
& \cdot\left[z_{i}\left(t_{k}\right)-z_{i}(t)+z_{i}(t)\right]+\sum_{j=1}^{n} b_{i j}(t) \\
& \left.\cdot\left[m_{j}\left(t_{k}\right) z_{j}\left(t_{k}\right)-m_{j}(t) z_{j}(t)+m_{j}(t) z_{j}(t)\right]\right\} \\
& =\sum_{i=1}^{n} \operatorname{sgn}\left(z_{i}(t)\right) \beta_{i}\left\{-a_{i}(t) e_{i}(t)+\sum_{j=1}^{n} b_{i j}(t) m_{j}\left(t_{k}\right)\right. \\
& \cdot e_{j}(t)-a_{i}(t) z_{i}(t)+b_{i i}(t) m_{i}\left(t_{k}\right) z_{i}(t) \\
& \left.+\sum_{j=1, j \neq i}^{n} b_{i j}(t) m_{j}\left(t_{k}\right) z_{j}(t)\right\} \leq \sum_{j=1}^{n}\left\{a_{j}(t)\right. \\
& \left.+\sum_{i=1}^{n} \frac{\beta_{i}}{\beta_{j}}\left|b_{i j}(t)\right| F_{j}\right\} \beta_{j}\left|e_{j}(t)\right| \\
& -\sum_{j=1}^{n}\left\{a_{j}(t)-b_{j j}^{+}(t) F_{j}-\sum_{i=1, i \neq j} \frac{\beta_{i}}{\beta_{j}}\left|b_{i j}(t)\right| F_{j}\right\} \\
& \cdot \beta_{j}\left|z_{j}(t)\right| \leq-N\|z(t)\|+M\|e(t)\| \leq-N\|z(t)\| \\
& +M \varphi(t)
\end{aligned}
$$

where $M$ is defined in (22), and

$$
m_{i}(t)= \begin{cases}\frac{h_{i}(t)}{z_{i}(t)}, & z_{i}(t) \neq 0 \\ 0, & z_{i}(t)=0\end{cases}
$$

On the other hand, by (43),

$$
\begin{aligned}
& \|z(t)\| \\
& \quad \leq\left\|z\left(t_{0}\right)\right\| \\
& \quad+\frac{1}{\Gamma(q)} \int_{t_{0}}^{t}(t-s)^{q-1}[-N\|z(s)\|+M \varphi(s)] d s
\end{aligned}
$$

$$
\begin{aligned}
= & \left\|z\left(t_{0}\right)\right\|-\frac{1}{\Gamma(q)} \int_{t_{0}}^{t}(t-s)^{q-1} N\|z(s)\| d s \\
& +\frac{1}{\Gamma(q)} \int_{t_{0}}^{t}(t-s)^{q-1} M \varphi(s) d s \\
\leq & \left\|z\left(t_{0}\right)\right\|-\frac{1}{\Gamma(q)} \int_{t_{0}}^{t}(t-s)^{q-1} N\|z(s)\| d s+M \delta,
\end{aligned}
$$

for $s \in\left[t_{k}, t\right), t \in\left[t_{k}, t_{k+1}\right)$, where $(1 / \Gamma(q)) \int_{t_{0}}^{t}(t-$ $s)^{q-1} \varphi(s) d s \leq \delta<+\infty$.

Using Lemma 3, from (45), it follows

$$
\begin{aligned}
\lim _{t \rightarrow+\infty}\|z(t)\| \leq \lim _{t \rightarrow+\infty} \Theta E_{q}\left(-N\left(t-t_{0}\right)^{q}\right)=0, & \\
& t \geq t_{0},
\end{aligned}
$$

where $\Theta=\left\|z\left(t_{0}\right)\right\|+M \delta$, which implies that $\|z(t)\|$ converges to 0 by the sampling time sequence $\left\{t_{k}\right\}_{k=0}^{+\infty}$. Therefore, system (6) reaches out-synchronization.

\subsection{Decentralized Data-Sampling Principle}

Theorem 7. Let $\phi(t)=\left(\phi_{1}(t), \phi_{2}(t), \ldots, \phi_{n}(t)\right)^{T}$ be positive and continuous on $\left[t_{0},+\infty\right)$. Set $t_{k+1}$ as a time point such that

$$
t_{k+1}=\sup _{\tau \geq t_{k}}\left\{\tau:\left|e_{i}(t)\right| \leq \phi_{i}(t), \forall t \in\left(t_{k}^{i}, \tau\right]\right\}
$$

for $i=1,2, \ldots, n$ and all $k=0,1,2, \ldots$, where $e(t)=$ $\left(e_{1}(t), e_{2}(t), \ldots, e_{n}(t)\right)^{T}$ is defined in (14). If there exist positive constants $\beta_{i}>0(i=1,2, \ldots, n)$ such that $\min _{1 \leq j \leq n} \lambda_{j}(\beta, t) \geq$ $K$ for some $K>0$ and all $t \geq t_{0}$, and $\sup _{t \geq t_{0}}(1 / \Gamma(q)) \int_{t_{0}}^{t}(t-$ $s)^{q-1}\|\phi(s)\| d s<+\infty$, then system (7) reaches outer-synchronization.

Proof. According to Lemma 2, from (13) and (47),

$$
\begin{gathered}
{ }^{C} D_{t_{k}}^{q}\|z(t)\|=\sum_{i=1}^{n} \beta_{i}{ }^{C} D_{t_{k}}^{q}\left|z_{i}(t)\right| \leq \sum_{i=1}^{n} \operatorname{sgn}\left(z_{i}(t)\right) \\
\cdot \beta_{i}{ }^{C} D_{t_{k}}^{q} z_{i}(t)=\sum_{i=1}^{n} \operatorname{sgn}\left(z_{i}(t)\right) \beta_{i}\left\{-a_{i}(t) z_{i}\left(t_{k}^{i}\right)\right. \\
\left.+\sum_{j=1}^{n} b_{i j}(t) h_{j}\left(t_{k}^{j}\right)\right\}=\sum_{i=1}^{n} \operatorname{sgn}\left(z_{i}(t)\right) \beta_{i}\left\{-a_{i}(t)\right. \\
\cdot\left[z_{i}\left(t_{k}^{i}\right)-z_{i}(t)+z_{i}(t)\right]+\sum_{j=1}^{n} b_{i j}(t)
\end{gathered}
$$




$$
\begin{aligned}
& \cdot\left[m_{j}\left(t_{k}^{j}\right) z_{j}\left(t_{k}^{j}\right)-m_{j}\left(t_{k}^{j}\right) z_{j}(t)\right. \\
& \left.\left.+m_{j}\left(t_{k}^{j}\right) z_{j}(t)\right]\right\}=\sum_{i=1}^{n} \operatorname{sgn}\left(z_{i}(t)\right) \beta_{i}\left\{-a_{i}(t)\right. \\
& \cdot e_{i}(t)-a_{i}(t) z_{i}(t)+b_{i i}(t) m_{i}\left(t_{k}^{i}\right) z_{i}(t) \\
& +\sum_{j=1}^{n} b_{i j}(t) m_{j}\left(t_{k}^{j}\right) e_{j}(t) \\
& \left.+\sum_{j=1, j \neq i}^{n} b_{i j}(t) m_{j}\left(t_{k}^{j}\right) z_{j}(t)\right\} \leq \sum_{j=1}^{n}\left\{a_{j}(t)\right. \\
& \left.+\sum_{i=1}^{n} \frac{\beta_{i}}{\beta_{j}}\left|b_{i j}(t)\right| F_{j}\right\} \beta_{j}\left|e_{j}(t)\right|-\sum_{j=1}^{n}\left\{a_{j}(t)\right. \\
& \left.+b_{j j}^{+}(t) F_{j}-\sum_{i=1, i \neq j} \frac{\beta_{i}}{\beta_{j}}\left|b_{i j}(t)\right| F_{j}\right\} \beta_{j}\left|z_{j}(t)\right| \\
& \leq K\|z(t)\|+M\|e(t)\| \leq-K\|z(t)\|+M\|\phi(t)\|,
\end{aligned}
$$

where $M$ is defined in (22), and

$$
m_{j}\left(t_{k}^{j}\right)= \begin{cases}\frac{h_{j}\left(t_{k}^{j}\right)}{z_{j}\left(t_{k}^{j}\right)}, & z_{j}\left(t_{k}^{j}\right) \neq 0, \\ 0, & z_{j}\left(t_{k}^{j}\right)=0 .\end{cases}
$$

On the other hand, by (48),

$$
\begin{aligned}
& \|z(t)\| \\
& \leq\left\|z\left(t_{0}\right)\right\| \\
& \quad+\frac{1}{\Gamma(q)} \int_{t_{0}}^{t}(t-s)^{q-1}[-K\|z(s)\|+M\|\phi(s)\|] d s \\
& =\left\|z\left(t_{0}\right)\right\|-\frac{1}{\Gamma(q)} \int_{t_{0}}^{t}(t-s)^{q-1} K\|z(s)\| d s \\
& \quad+\frac{1}{\Gamma(q)} \int_{t_{0}}^{t}(t-s)^{q-1} M\|\phi(s)\| d s \\
& \leq\left\|z\left(t_{0}\right)\right\|-\frac{1}{\Gamma(q)} \int_{t_{0}}^{t}(t-s)^{q-1} K\|z(s)\| d s+M \rho,
\end{aligned}
$$

for $s \in\left[t_{k}^{i}, t\right), t \in\left[t_{k}^{i}, t_{k+1}^{i}\right)$, where $(1 / \Gamma(q)) \int_{t_{0}}^{t}(t-$ $s)^{q-1}\|\phi(s)\| d s \leq \rho<+\infty$.

Using Lemma 3, from (50), it follows

$$
\lim _{t \rightarrow+\infty}\|z(t)\| \leq \lim _{t \rightarrow+\infty} \Pi E_{q}\left(-K\left(t-t_{0}\right)^{q}\right)=0, \quad t \geq t_{0},
$$

where $\Pi=\left\|z\left(t_{0}\right)\right\|+M \rho$, which implies that $\|z(t)\|$ converges to 0 by the sampling time sequence $\left\{t_{k}^{i}\right\}_{k=0}^{+\infty}, i=1,2, \ldots, n$. Therefore, system (7) reaches out-synchronization.

Remark 8. As Theorem 5 in [19], under the data-sampling rule in Theorem 6 or Theorem 7, the interevent interval of each system state is strictly positive and possesses a common positive lower bound. Furthermore, the Zeno behavior is excluded.

Remark 9. For the sampled-data control, how to choose the proper scheme with the ultimate aim of maximizing the data collected to control the system is challenging. For example, as revealed in $[9,10]$, it is extremely difficult to design the sampling time point inherited from the sampled-data control strategy. However, according to Theorems 4-7, this situation can be effectively solved if the centralized and decentralized data-sampling principles are cleverly utilized.

Remark 10. For three control schemes in Theorems 4-7, these are just the type and level of points, not the merits of good points of difference. Theorem 4 is entirely focused around the centralized data-sampling principle via structure. Theorem 6 is concerned with the centralized data-sampling principle via state. Theorem 7 is to place emphasis on the decentralized data-sampling principle via state.

Remark 11. Note that the sampled-data control in Theorems 4-7 exerts only at the sampling time point, that is, every system state employs only its neighbors' information at $t_{k}$ or $t_{k}^{i}$. Thus, compared with the continuous-time control strategy, the control schemes in Theorems 4-7 can effectively save the bandwidth and reduce the communication cost. Moreover, the results obtained here are the first ones on centralized and decentralized data-sampling principles for outer-synchronization of fractional-order neural networks.

Remark 12. The key features of outer-synchronization in Theorems 4-7 are follows. (1) Each outer-synchronization scheme is closely related to the sampling time point. Once the sampling time point is given, the states of the controlled fractional-order neural networks will achieve outersynchronization. (2) Centralized data-sampling principle via structure makes full use of the characteristic of system itself, while centralized or decentralized data-sampling principle via state skillfully combines the feature of state measurement error.

Remark 13. The analytical methods for outer-synchronization in Theorems 4-7 are quite different from conventional complete synchronization, projective synchronization, phase synchronization, distributed synchronization, pinning synchronization, and cluster synchronization.

\section{A Numerical Example}

In this section, a numerical example is utilized to show the effectiveness of the results obtained. 




FIGURE 1: Dynamics of $u_{1}(t)$ and $v_{1}(t)$ in the triggering mechanism as Theorem 4.

Consider a class of fractional-order neural networks as follows:

$$
\begin{aligned}
{ }^{C} D_{t_{0}}^{q} x_{1}(t)= & -a_{1}(t) x_{1}(t)+b_{11}(t) f_{1}\left(x_{1}(t)\right) \\
& +b_{12}(t) f_{2}\left(x_{2}(t)\right)+u_{1}(t), \\
{ }^{C} D_{t_{0}}^{q} x_{2}(t)= & -a_{2}(t) x_{2}(t)+b_{21}(t) f_{1}\left(x_{1}(t)\right) \\
& +b_{22}(t) f_{2}\left(x_{2}(t)\right)+u_{2}(t),
\end{aligned}
$$

where $q=1 / 2, t_{0}=0, A=\left(\begin{array}{cc}a_{1}(t) & 0 \\ 0 & a_{2}(t)\end{array}\right)=\left(\begin{array}{cc}1.5 & 0 \\ 0 & 1.5\end{array}\right), B=$ $\left(\begin{array}{l}b_{11}(t) b_{12}(t) \\ b_{21}(t) \\ b_{22}(t)\end{array}\right)=\left(\begin{array}{cc}0.3 & -0.7 \\ -1.2 & -0.1\end{array}\right), u=\left(\begin{array}{l}u_{1}(t) \\ u_{2}(t)\end{array}\right)=\left(\begin{array}{l}0.1 \\ 0.1\end{array}\right), f_{1}(\mathbb{X})=$ $f_{2}(\mathbb{X})=1 /\left(1+e^{-囚}\right)$.

By direct calculation, we can obtain

$$
\begin{aligned}
& \lambda_{1}(\beta, t)=a_{1}(t)-F_{1} b_{11}^{+}(t)-\frac{F_{1} \beta_{2}}{\beta_{1}}\left|b_{21}(t)\right|, \\
& \lambda_{2}(\beta, t)=a_{2}(t)-F_{2} b_{22}^{+}(t)-\frac{F_{2} \beta_{1}}{\beta_{2}}\left|b_{12}(t)\right| .
\end{aligned}
$$

To choose $\varepsilon=0.2, \iota=0.8, \beta_{1}=\beta_{2}=1$, then it follows that $B=1.6, C=1.05$. Hence the following inequalities hold:

$$
\begin{aligned}
B \varepsilon-\iota & \leq 0, \\
C \varepsilon-\iota(2-\varepsilon) & \leq 0 .
\end{aligned}
$$

According to Theorem 4, system (52) reaches outer-synchronization. Figures 1 and 2 depict the dynamics of $u_{1}(t)$ and $v_{1}(t), u_{2}(t)$ and $v_{2}(t)$ in the triggering time points as Theorem 4, respectively. Figure 3 describes the release time points and release intervals.

To select $\varphi(t)=1 /(t+1)^{1 / 2}$, together with

$$
\sup _{t \geq 0} \frac{1}{\Gamma(1 / 2)} \int_{0}^{t}(t-s)^{q-1} \frac{1}{(s+1)^{1 / 2}} d s<+\infty
$$



Figure 2: Dynamics of $u_{2}(t)$ and $v_{2}(t)$ in the triggering mechanism as Theorem 4.

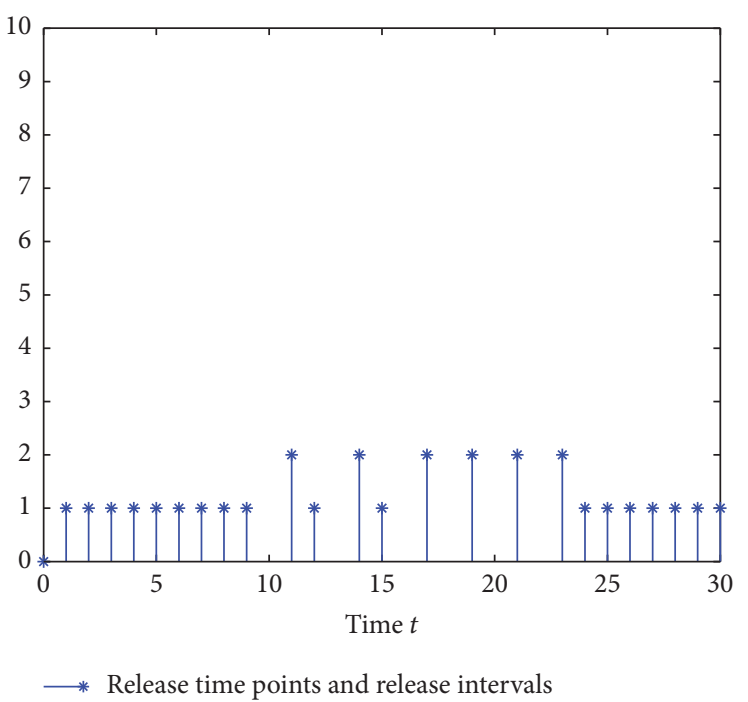

FIGURE 3: The release time points and release intervals in the triggering mechanism as Theorem 4 .

according to Theorem 6, system (52) reaches outer-synchronization. Figures 4 and 5 depict the dynamics of $u_{1}(t)$ and $v_{1}(t), u_{2}(t)$ and $v_{2}(t)$ in the triggering time points as Theorem 6 , respectively. Figure 6 describes the release time points and release intervals.

To select $\phi_{1}(t)=1 /(t+1)^{1 / 2}, \phi_{2}(t)=1 /(t+2)^{1 / 2}$, together with

$$
\begin{aligned}
& \sup _{t \geq 0} \frac{1}{\Gamma(1 / 2)} \int_{0}^{t}(t-s)^{q-1} \frac{1}{(s+1)^{1 / 2}} d s<+\infty \\
& \sup _{t \geq 0} \frac{1}{\Gamma(1 / 2)} \int_{0}^{t}(t-s)^{q-1} \frac{1}{(s+2)^{1 / 2}} d s<+\infty
\end{aligned}
$$




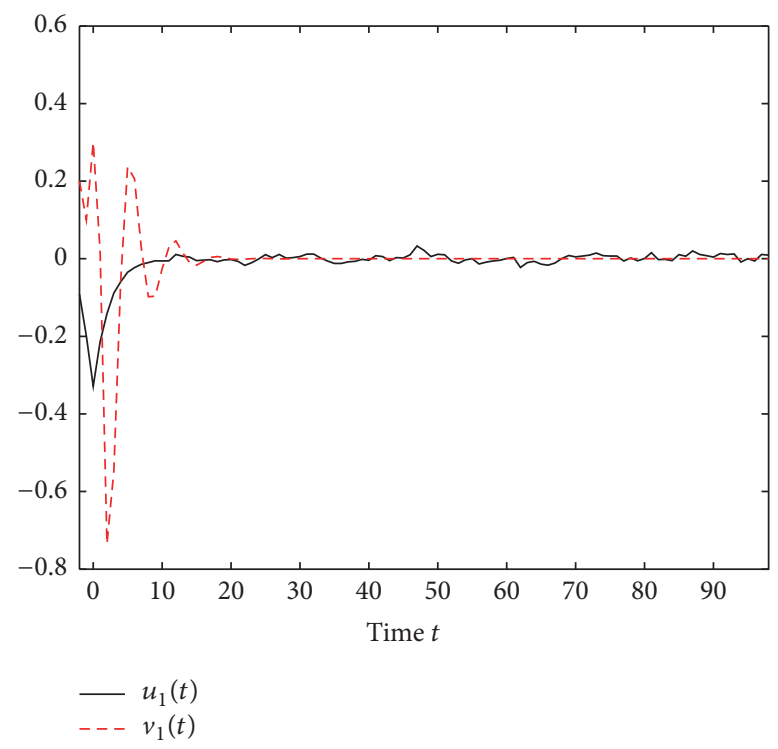

FIgURE 4: Dynamics of $u_{1}(t)$ and $v_{1}(t)$ in the triggering mechanism as Theorem 6 .

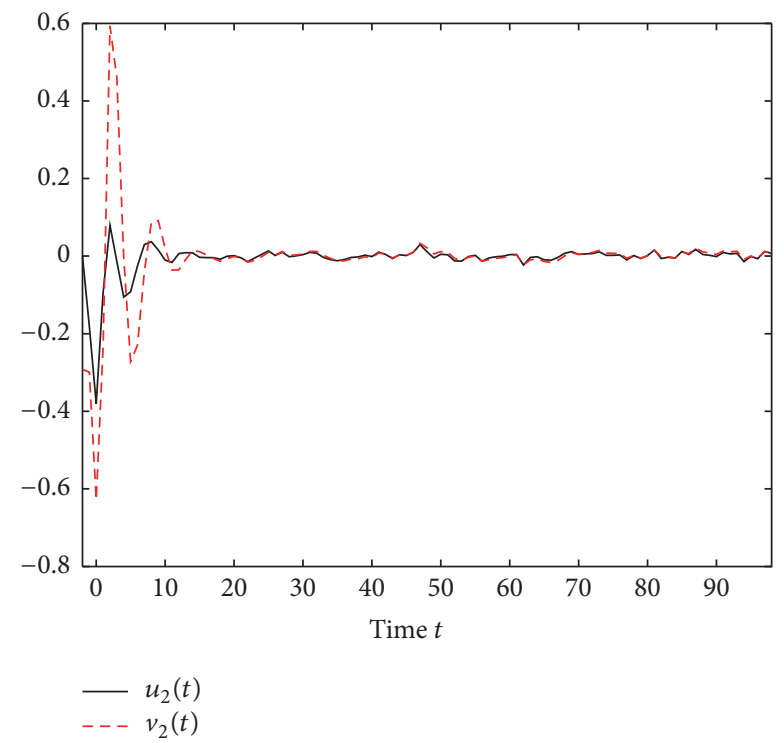

FIgURe 5: Dynamics of $u_{2}(t)$ and $v_{2}(t)$ in the triggering mechanism as Theorem 6

according to Theorem 7, system (52) reaches outersynchronization. Figures 7 and 8 depict the dynamics of $u_{1}(t)$ and $v_{1}(t), u_{2}(t)$ and $v_{2}(t)$ in the triggering time points as Theorem 7 , respectively. Figure 9 describes the release time points and release intervals.

Remark 14. In existing publications, there has been no theoretic criterion to achieve outer-synchronization of (52). In addition, using centralized or decentralized data-sampling principle to analyze and control fractional-order systems is also rare.

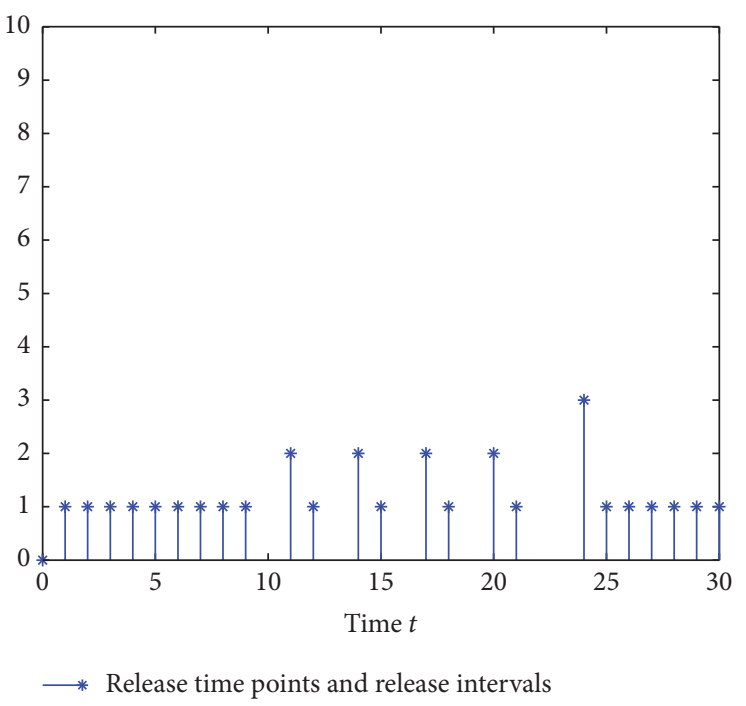

Figure 6: The release time points and release intervals in the triggering mechanism as Theorem 6 .

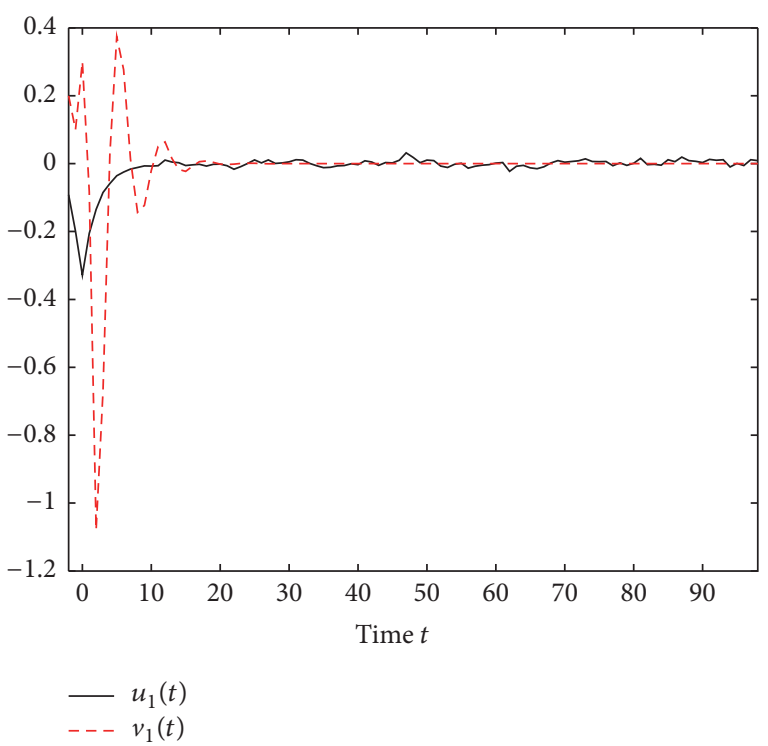

FIGURE 7: Dynamics of $u_{1}(t)$ and $v_{1}(t)$ in the triggering mechanism as Theorem 7 .

Remark 15. According to simulation analysis in Figures $1-9$, there is no essential difference regarding outersynchronization performance in three control schemes as Theorems 4-7. By comparative analysis of Figures 3, 6, and 9 , the release intervals via control scheme as Theorem 4 are relatively minor, and the triggering time points via control scheme as Theorem 7 are spread more thinly.

\section{Concluding Remarks}

In this paper, we show that outer-synchronization of fractional-order neural networks can be achieved by applying appropriate centralized and decentralized data-sampling 


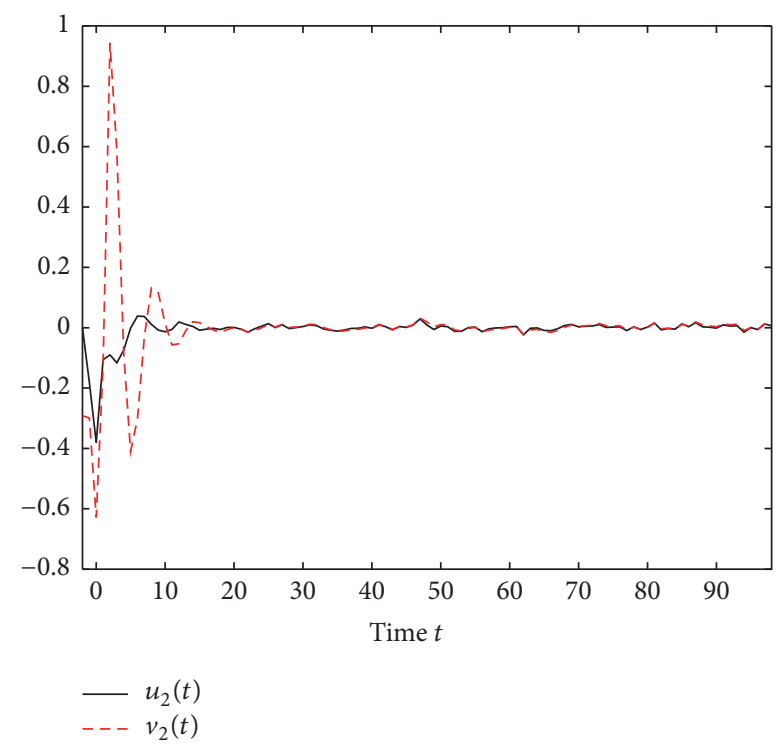

Figure 8: Dynamics of $u_{2}(t)$ and $v_{2}(t)$ in the triggering mechanism as Theorem 7.



FIGURE 9: The release time points and release intervals in the triggering mechanism as Theorem 7 .

principles. Such theoretical results improve and supplement some existing related results. The results obtained here are sufficient conditions for outer-synchronization of fractional-order neural networks and may remain room for improvement. Further extensions would be welcome: (1) outer-synchronization of fractional-order neural networks considering both conservativeness and complexity; (2) analyzing the outer-synchronization of fractional-order neural networks subject to time-delay; (3) analyzing the outersynchronization of fractional-order neural networks subject to stochastic disturbance.

\section{Conflicts of Interest}

The author declares that there are no conflicts of interest regarding the publication of this paper.

\section{Acknowledgments}

The work is supported by the Research Project of Hubei Provincial Department of Education of China under Grant T201412.

\section{References}

[1] B. Chen and J. Chen, "Global asymptotical $\omega$-periodicity of a fractional-order non-autonomous neural networks," Neural Networks, vol. 68, pp. 78-88, 2015.

[2] I. Pan and S. Das, "Fractional order AGC for distributed energy resources using robust optimization," IEEE Transactions on Smart Grid, vol. 7, no. 5, pp. 2175-2186, 2015.

[3] J. Shen and J. Lam, "Stability and performance analysis for positive fractional-order systems with time-varying delays," IEEE Transactions on Automatic Control, vol. 61, no. 9, pp. 26762681, 2016

[4] L. P. Chen, R. C. Wu, J. Cao, and J.-B. Liu, "Stability and synchronization of memristor-based fractional-order delayed neural networks," Neural Networks, vol. 71, pp. 37-44, 2015.

[5] C. Huang, J. Cao, M. Xiao, A. Alsaedi, and T. Hayat, "Bifurcations in a delayed fractional complex-valued neural network," Applied Mathematics and Computation, vol. 292, pp. 210-227, 2017.

[6] S. Liang, R. Wu, and L. Chen, "Comparison principles and stability of nonlinear fractional-order cellular neural networks with multiple time delays," Neurocomputing, vol. 168, pp. 618625, 2015.

[7] M. B. Delghavi, S. Shoja-Majidabad, and A. Yazdani, "Fractional-order sliding-mode control of islanded distributed energy resource systems," IEEE Transactions on Sustainable Energy, vol. 7, no. 4, pp. 1482-1491, 2016.

[8] R. Rakkiyappan, G. Velmurugan, and J. Cao, "Stability analysis of fractional-order complex-valued neural networks with time delays," Chaos, Solitons and Fractals, vol. 78, pp. 297-316, 2015.

[9] A. Wu, L. Liu, T. Huang, and Z. Zeng, "Mittag-Leffler stability of fractional-order neural networks in the presence of generalized piecewise constant arguments," Neural Networks, vol. 85, pp. 118-127, 2017.

[10] A. Wu and Z. Zeng, "Boundedness, Mittag-Leffler stability and asymptotical $\omega$-periodicity of fractional-order fuzzy neural networks," Neural Networks, vol. 74, pp. 73-84, 2016.

[11] A. L. Wu and Z. G. Zeng, "Global Mittag-Leffler stabilization of fractional-order memristive neural networks," IEEE Transactions on Neural Networks and Learning Systems, vol. 28, no. 1, pp. 206-217, 2017.

[12] X. Yang, C. Li, Q. Song, T. Huang, and X. Chen, "Mittag-Leffler stability analysis on variable-time impulsive fractional-order neural networks," Neurocomputing, vol. 207, pp. 276-286, 2015.

[13] N. Ullah, M. Asghar Ali, R. Ahmad, and A. Khattak, "Fractional order control of static series synchronous compensator with parametric uncertainty," IET Generation, Transmission \& Distribution, vol. 11, no. 1, pp. 289-302, 2017. 
[14] S. Zhang, Y. G. Yu, and H. Wang, "Mittag-Leffler stability of fractional-order Hopfield neural networks," Nonlinear Analysis: Hybrid Systems, vol. 16, pp. 104-121, 2015.

[15] A. Azami, S. V. Naghavi, R. Dadkhah Tehrani, M. H. Khooban, and F. Shabaninia, "State estimation strategy for fractional order systems with noises and multiple time delayed measurements," IET Science, Measurement \& Technology, vol. 11, no. 1, pp. 9-17, 2017.

[16] T. Jing, F. Chen, and Q. Li, "Finite-time mixed outer synchronization of complex networks with time-varying delay and unknown parameters," Applied Mathematical Modelling, vol. 39, no. 23-24, pp. 7734-7743, 2015.

[17] S. Li, "Linear generalized outer synchronization between two complex dynamical networks with time-varying coupling delay," Optik, vol. 127, no. 22, pp. 10467-10477, 2016.

[18] J. Lu, C. Ding, J. Lou, and J. Cao, "Outer synchronization of partially coupled dynamical networks via pinning impulsive controllers," Journal of the Franklin Institute. Engineering and Applied Mathematics, vol. 352, no. 11, pp. 5024-5041, 2015.

[19] W. Lu, R. Zheng, and T. Chen, "Centralized and decentralized global outer-synchronization of asymmetric recurrent timevarying neural network by data-sampling," Neural Networks, vol. 75, pp. 22-31, 2016.

[20] W. G. Sun, Y. Q. Wu, J. Y. Zhang, and S. Qin, "Inner and outer synchronization between two coupled networks with interactions," Journal of the Franklin Institute, vol. 352, no. 8, pp. 3166-3177, 2015.

[21] Y. Sun, W. Li, and J. Ruan, "Generalized outer synchronization between complex dynamical networks with time delay and noise perturbation," Communications in Nonlinear Science and Numerical Simulation, vol. 18, no. 4, pp. 989-998, 2013.

[22] Z. Wu, G. Chen, and X. Fu, "Outer synchronization of driveresponse dynamical networks via adaptive impulsive pinning control," Journal of the Franklin Institute, vol. 352, no. 10, pp. 4297-4308, 2015.

[23] Y. Yang, Y. Wang, and T. Li, "Outer synchronization of fractional-order complex dynamical networks," Optik, vol. 127, no. 19, pp. 7395-7407, 2016.

[24] W.-H. Chen, Z. Wang, and X. Lu, "On sampled-data control for master-slave synchronization of chaotic Lur'e systems," IEEE Transactions on Circuits and Systems II: Express Briefs, vol. 59, no. 8, pp. 515-519, 2012.

[25] W.-H. Chen and W. X. Zheng, "An improved stabilization method for sampled-data control systems with control packet loss," IEEE Transactions on Automatic Control, vol. 57, no. 9, pp. 2378-2384, 2012.

[26] D. Ding, Z. Wang, G. Wei, and F. E. Alsaadi, "Event-based security control for discrete-time stochastic systems," IET Control Theory \& Applications, vol. 10, no. 15, pp. 1808-1815, 2016.

[27] H. Li, X. Liao, T. Huang, and W. Zhu, "Event-triggering sampling based leader-following consensus in second-order multi-agent systems," IEEE Transactions on Automatic Control, vol. 60, no. 7, pp. 1998-2003, 2015.

[28] D. Wang, D. R. Liu, Q. C. Zhang, and D. B. Zhao, "Data-based adaptive critic designs for nonlinear robust optimal control with uncertain dynamics," IEEE Transactions on Systems, Man, and Cybernetics: Systems, vol. 46, no. 11, pp. 1544-1555, 2016.

[29] J. Wang, X.-M. Zhang, and Q.-L. Han, "Event-triggered generalized dissipativity filtering for neural networks with timevarying delays," IEEE Transactions on Neural Networks and Learning Systems, vol. 27, no. 1, pp. 77-88, 2015.
[30] L. C. Wang, Z. D. Wang, T. W. Huang, and G. L. Wei, "An eventtriggered approach to state estimation for a class of complex networks with mixed time delays and nonlinearities," IEEE Transactions on Cybernetics, vol. 46, no. 11, pp. 2497-2508, 2016.

[31] Z. Wang and D. Liu, "A data-based state feedback control method for a class of nonlinear systems," IEEE Transactions on Industrial Informatics, vol. 9, no. 4, pp. 2284-2292, 2013.

[32] S. Wen, T. Huang, X. Yu, M. Z. Chen, and Z. Zeng, "Aperiodic sampled-data sliding-mode control of fuzzy systems with communication delays via the event-triggered method," IEEE Transactions on Fuzzy Systems, vol. 24, no. 5, pp. 1048-1057, 2016.

[33] H.-Q. Xiao, Y. He, M. Wu, S.-P. Xiao, and J. She, "New results on Ho tracking control based on the T-S fuzzy model for sampleddata networked control system," IEEE Transactions on Fuzzy Systems, vol. 23, no. 6, pp. 2439-2448, 2015.

[34] C.-K. Zhang, Y. He, and M. Wu, "Improved global asymptotical synchronization of chaotic lur'e systems with sampled-data control," IEEE Transactions on Circuits and Systems II: Express Briefs, vol. 56, no. 4, pp. 320-324, 2009.

[35] H. Zhang, J. Liu, D. Ma, and Z. Wang, "Data-core-based fuzzy min-max neural network for pattern classification," IEEE Transactions on Neural Networks, vol. 22, no. 12, pp. 2339-2352, 2011.

[36] X. X. Yin, D. Yue, S. L. Hu, C. Peng, and Y. S. Xue, "Model-based event-triggered predictive control for networked systems with data dropout," SIAM Journal on Control and Optimization, vol. 54, no. 2, pp. 567-586, 2016.

[37] X.-M. Zhang and Q.-L. Han, "Event-triggered dynamic output feedback control for networked control systems," IET Control Theory \& Applications, vol. 8, no. 4, pp. 226-234, 2014.

[38] R. Zheng, X. Yi, W. Lu, and T. Chen, "Stability of analytic neural networks with event-triggered synaptic feedbacks," IEEE Transactions on Neural Networks and Learning Systems, vol. 27, no. 2, pp. 483-494, 2016.

[39] H. Ye, J. Gao, and Y. Ding, "A generalized Gronwall inequality and its application to a fractional differential equation," Journal of Mathematical Analysis and Applications, vol. 328, no. 2, pp. 1075-1081, 2007. 




Advances in

Operations Research

vatem alat4

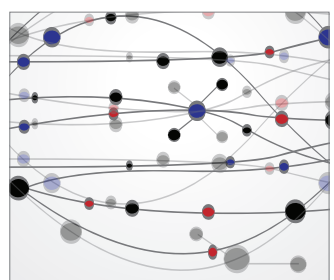

\section{The Scientific} World Journal
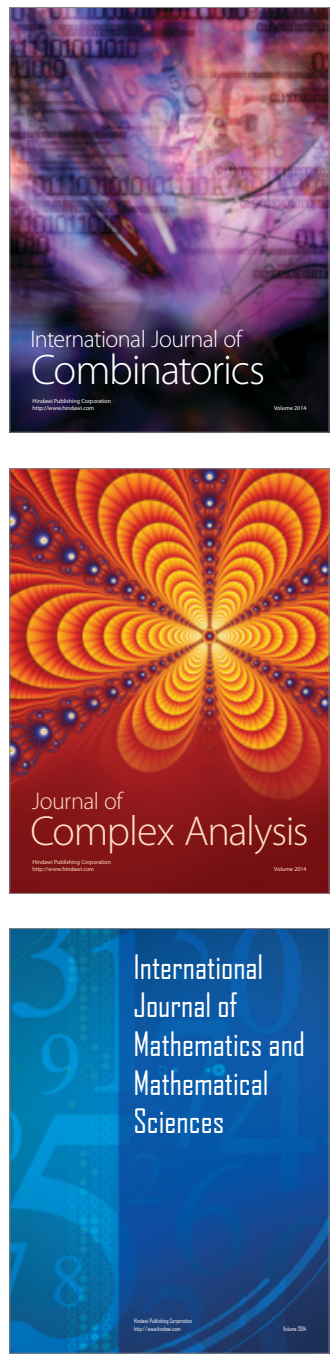
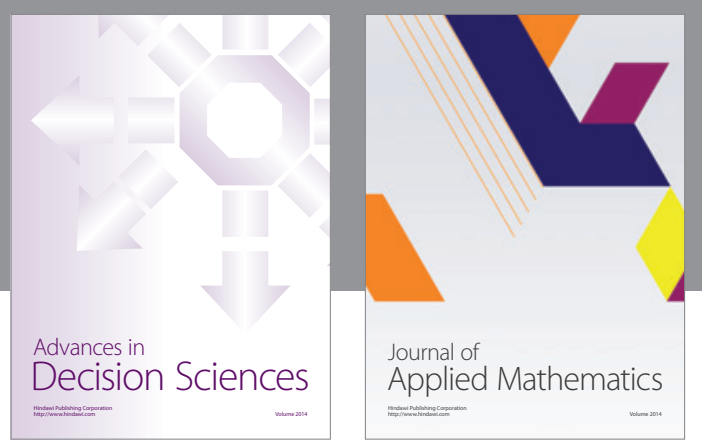

Algebra

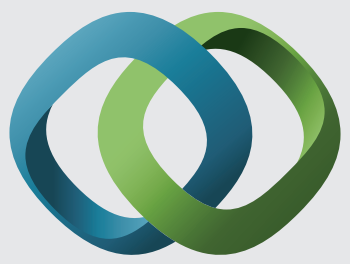

\section{Hindawi}

Submit your manuscripts at

https://www.hindawi.com
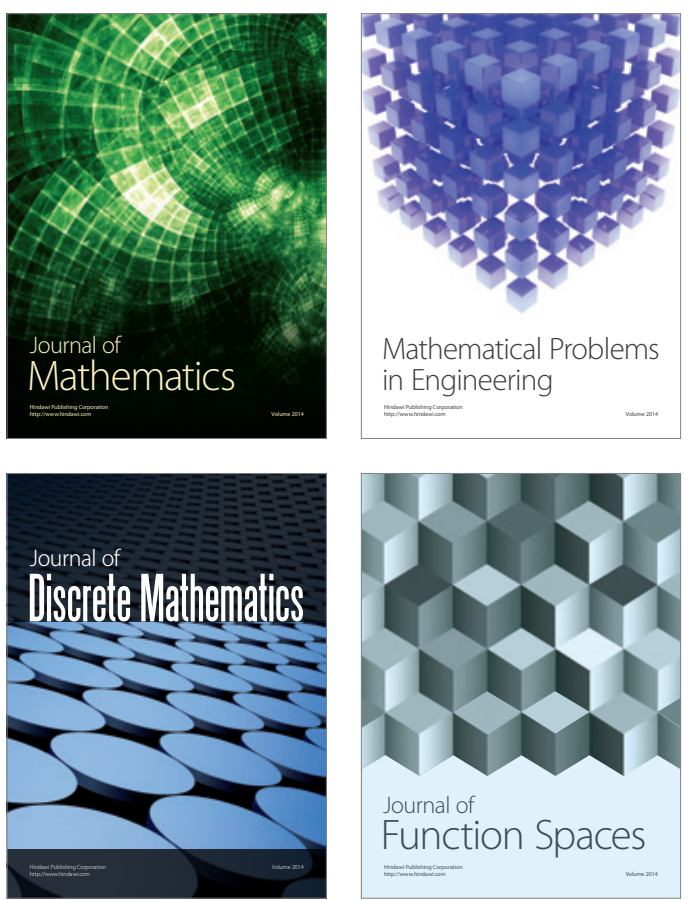

Mathematical Problems in Engineering
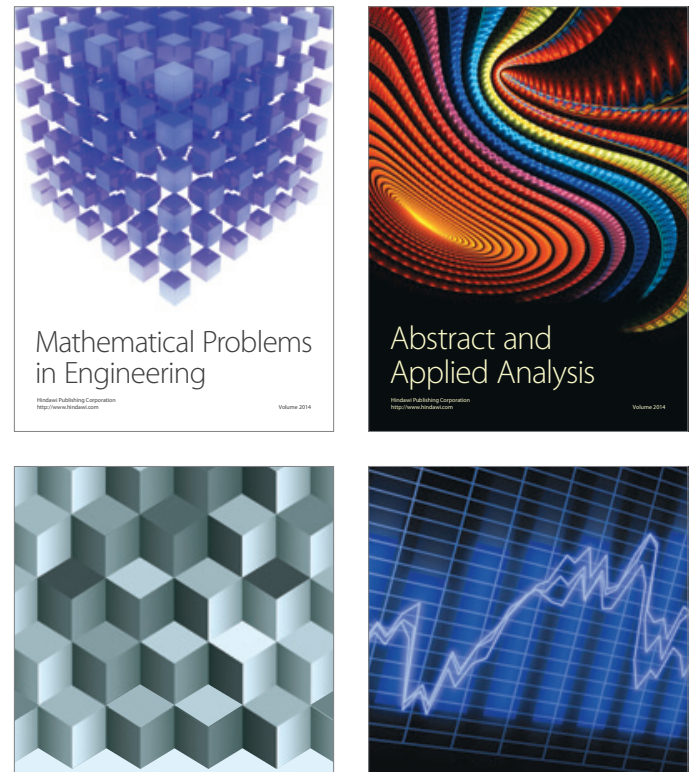

Journal of

Function Spaces

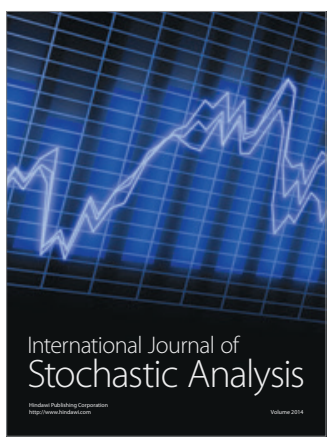

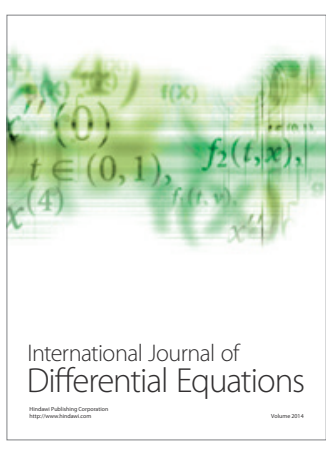
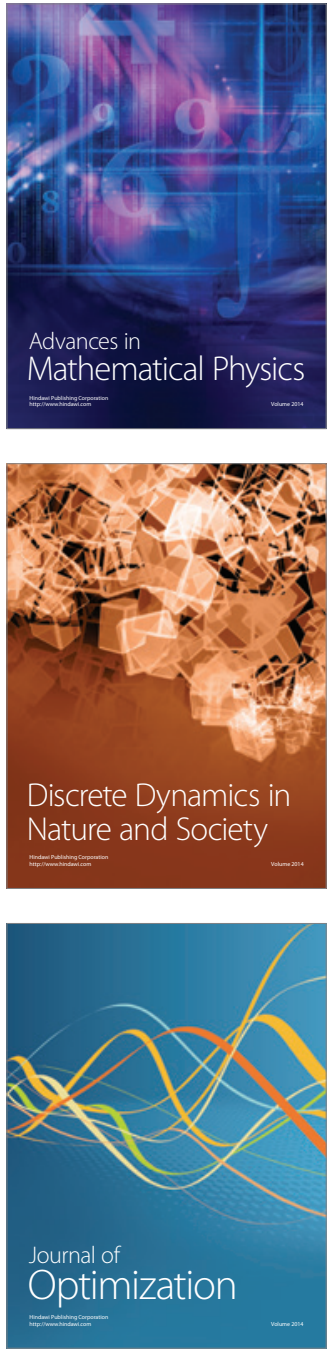